\title{
DANIEL GARCÍA SAN JOSÉ: RIESGOS EPIGENÉTICOS DE LA GESTACIÓN POR SUSTITUCIÓN PARA LA SALUD DE LOS NIÑOS ASÍ CONCEBIDOS: ¿QUIÉN VELA POR EL INTERÉS SUPERIOR DEL MENOR?
}

\author{
EPIGENETIC RISKS IN THE COMMERCIAL SURROGACY FOR THE CHILDREN'S \\ HEALTH SO CONCEIVED. WHO CARES THE SUPERIOR INTEREST OF THE \\ MINOR?
}

\section{RESUMEN}

Esta investigación socio-jurídica que cuenta con el respaldo de un proyecto de excelencia financiado por el MINECO en su convocatoria RETOS de 2015 (DER2015-64151-R) se ha hecho desde una aproximación de Derecho internacional y europeo siguiendo una metodología científica denominada desk table research que implica una relectura del método positivista-sociológico desde un prisma postmoderno y en la que se combinan diversas técnicas metodológicas (estudio de casos concretos y examen de la doctrina relevante) a fin de defender la necesidad de contar con una regulación internacional para la gestación por sustitución inexistente en la actualidad- desde la protección internacional de los derechos humanos y en especial, asumiendo como principio rector el interés superior del menor. Salvo error por nuestra parte, hasta la fecha no se ha considerado la epigenética al hablar del interés superior del menor en el contexto de la gestación por sustitución, de ahí la pertinencia y el carácter novedoso del análisis que desarrollamos centrado en el examen de los riesgos epigenéticos derivados de la gestación por sustitución para la salud del niño así concebido.

\section{ABSTRACT}

This socio-legal research supported with public funds (MINECO, DER2015-64151-R) is made from an approximation of international and European law following a scientific methodology called desk table research that involves rereading the positivist-sociological method from a postmodern prism and in which various methodological techniques (study of specific cases and review of the relevant doctrine) are combined to defend the need for international regulation for commercial surrogacy -not yet in force- from the international protection of human rights approach and taking as its guiding principle the best interests of the child. Except error on our part, so far epigenetics has not been considered when speaking of the best interests of the child in the context of commercial surrogacy, hence the 
relevance and novelty of the analysis we develop focused on the examination of the epigenetic risks implied in commercial surrogacy for the health of children thus conceived.

PALABRAS CLAVE: Implicaciones éticas y legales de la epigenética - Origen epigenético de enfermedades asociadas a la edad adulta - Contratos de gestación por sustitución - Interés superior del menor

KEY WORDS: Legal and Ethical Implications of Epigenetics - Epigenetics in Human Disease - Commercial Surrogacy Arrangements - Best Interest of the Minor

\section{INTRODUCCIÓN}

Los contratos privados de gestación por sustitución son hoy día un negocio global, que mueve billones de dólares anualmente y al que nadie se atreve a poner límites (Sánchez Morales, 2011, 765). Desde su aparición, hace décadas, vienen planteando problemas jurídicos de hondo calado para los Estados-no sólo desde el punto del Derecho Civil y de la filiación- sino especialmente desde consideraciones éticas y jurídicas (Drabiak, 2007, 300-309; Deonandan, 2012, 742; Ettinger, 2012, 243-268). Pese a ello, aún no existe una regulación internacional al respecto, ante la falta de consenso entre los Estados que conforman la comunidad internacional.

En esta investigación partimos de una doble premisa: de una parte, la práctica de la gestación por sustitución es una realidad y por tanto, el Derecho, como herramienta reguladora de las acciones sociales, debe asumir una postura determinada frente a ésta. De otra parte, está la evidencia de los últimos años, de que los problemas jurídicos de la gestación por sustitución no se resuelven dejando las cosas como están. Partiendo de esta doble premisa, el objetivo que hemos perseguimos en nuestra investigación ha sido responder a la pregunta de cuál es la mejor aproximación del Derecho a esta cuestión. A tal fin, pensamos que es necesario considerar los riesgos epigenéticos derivados para la salud de los niños nacidos mediante esta técnica de reproducción asistida, asumiendo que salvo error por nuestra parte esta aproximación novedosa no se ha realizado hasta la fecha en la Doctrina jurídica.

\section{MATERIAL Y MÉTODOS}

Esta investigación socio-jurídica que cuenta con el respaldo de un proyecto de excelencia financiado por el MINECO en su convocatoria RETOS de 2015 (DER2015-64151-R) se ha hecho desde una aproximación de Derecho internacional y europeo siguiendo una metodología científica denominada desk table research que implica una relectura del método positivista-sociológico desde un prisma 
postmoderno y en la que se combinan diversas técnicas metodológicas: estudio de casos concretos, examen de la doctrina relevante, para recoger información genérica, identificar problemas clave, sus posibles soluciones así como las posibles lagunas existentes a nivel de investigación (Reed y Padkocimate, 2012, 2); estadísticas y otros estudios sociológicos aunque con suficiente cautela considerando sus limitaciones en el campo de las ciencias jurídicas (Landman y Carvahlo, 2010, 106); análisis crítico-jurídico de la normativa existente identificando lagunas y deficiencias y proponiendo de lege ferenda propuestas válidas para afrentar los riesgos epigenéticos en relación con la gestación por sustitución.

\section{DISCUSIÓN}

La epigenética es una vieja conocida de la comunidad científica desde que fuera mencionado por primera vez en 1942 por Conrad Waddington, describiendo las interacciones entre los genes y su entorno. La epigenética indica cambios heredables en la estructura y organización del ADN que aunque no suponen una alteración de la secuencia de los nucleótidos, modifican la expresión génica y conllevan cambios heredables en el fenotipo (García Robles, 2012, 61). Dicho de otro modo, las condiciones de vida que experimente una persona (por ejemplo, si vivió en un ambiente con mayor o menor salubridad o si desarrolló unos hábitos vitales más o menos saludables) de algún modo se quedan archivadas en un "disco duro de memoria" que -de un modo poco conocido aún por los científicosdeterminan años más tarde una respuesta somática -epimutaciones- (por ejemplo, el desarrollo de enfermedades directamente conectadas a tales condiciones) en esas personas y en sus descendientes aun cuando éstos últimos no las hayan experimentado directamente por sí mismos.

Dos de las características de los cambios epigenéticos o epimutaciones son de especial relevancia para el caso de la gestación por sustitución: el carácter transmisible de las epimutaciones (de padres a hijos) y el carácter variable de las epimutaciones según la etapa de desarrollo de cada individuo. La importancia del primer dato es evidente: en la gestación por sustitución las epimutaciones del bebé así concebido no van a depender tanto de la carga genética de los padres de intención, en el supuesto en que ambos hayan aportado sus gametos (gestación por sustitución parcial o gestational surrogacy), o si es sólo el esperma del padre de intención el introducido en la gestante para fertilizar su óvulo (gestación por subrogación plena o complete surrogacy), como de la carga epigenética de la mujer que lo gesta por sustitución y que biológicamente será la madre del nasciturus al alumbrarlo (mater semper certa est), con independencia de que legalmente no lo sea en virtud de un contrato privado inter partes -oficializado por una sentencia judicial 
en un litigio amistoso, por el que renuncia a sus derechos como madre sobre su bebé en favor de los padres de intención.

Esta afirmación vendría a inferirse de las evidencias aportadas en recientes estudios, que concluyen que las epimutaciones son inducidas entre otros estímulos por los cuidados maternos o por el estrés de la madre durante la gestación o inmediatamente después del parto (Gallou-Kabani, 2007, 321-323; Brennan y Capel, 2004, 510. Dolinoy, Weidman y Jirtle, 2007, 298).

Aún es mayor la relevancia del segundo dato que hemos destacado -el carácter variable de las epimutaciones según la etapa de desarrollo de cada individuo- a propósito del carácter transmisible de las epimutaciones en los contratos de gestación por subrogación. En este punto los autores coinciden en señalar que la edad a la que un organismo se expone a las epimutaciones es determinante para cuantificar el grado de incidencia de dicha epimutación, de manera que la etapa de gestación del feto y los meses posteriores al alumbramiento son los momentos de desarrollo del ser humano en los que éste resulta más proclive a experimentar dichas epimutaciones. Como han señalado Rothstein, Cai y Marchant $(2009,5)$, la epigenética proporciona un mecanismo para el ser humano en formación -en el útero o en los primeros estados de vida tras nacer- cuya misión es evaluar su entorno y ajustar su respuesta genética al mismo.

En un continuo proceso de retroalimentación o "feedback", el medio ambiente, la dieta, estilo de vida, estrés maternal, entre otros factores, permiten a un organismo en desarrollo ajustar su fenotipo (la expresión de sus genes) a su entorno anticipado, de manera que la epigenética actúa como un mediador entre el medio ambiente y el genoma. Ahora bien, esa adaptación de la expresión genómica del ser humano en formación al entorno se ve alterada de manera dramática en el caso de la gestación por sustitución pues en casi todos los supuestos se trata de un bebé "encargado" por padres de países desarrollados (europeos fundamentalmente) a mujeres gestantes en países en desarrollo (estando la India a la cabeza) con unas condiciones ambientales, de dieta, de hábitos de vida, etc. muy diferentes con las que se van a encontrar esos niños una vez sean entregado a sus padre de intención y para las cuáles no han sido predispuestos por las epimutaciones transmitidas por su madre biológica. Su entorno natural va a cambiar significativamente en un momento de su desarrollo celular en el que más fácilmente pueden producirse epimutaciones que pueden dar lugar a enfermedades a desarrollar en su estado adulto -y que además, transmitirá a sus descendientes-. Se trata de enfermedades para las que su organismo en principio no estaría expuesto de haber seguido viviendo en el entorno ambiental, económico y social de su madre biológica.

Así parece deducirse de los trabajos de autores como Andrew Feinberg quien sostiene -siguiendo un razonamiento lógico- que si las epimutaciones pueden 
explicar un desarrollo normal de un ser humano en un entorno concreto, de igual manera, las interrupciones en las epimutaciones deben alterar necesariamente los programas normales de desarrollo de ese mismo ser humano (lo que se conoce como aberraciones epigenéticas) pudiendo comprometer negativamente su salud y bienestar (Feinberg, 2008, 1348).

En efecto, cada vez está más aceptado entre la comunidad científica el hecho de que la predisposición a ciertas enfermedades manifestadas en la etapa adulta de una persona tienen su origen en epimutaciones originadas durante su etapa embrionaria. Siguiendo la teoría conocida como "early origins" (orígenes tempranos) según la cual la evolución de la plasticidad del desarrollo que permite que un organismo pueda adaptarse a las señales ambientales durante la vida temprana, también puede aumentar el riesgo de desarrollar enfermedades crónicas cuando hay un desajuste entre lo percibido en los primeros estados de desarrollo de la persona y lo que va a encontrar realmente en su etapa adulta (Jirtle y Skinner, 2007, 253).

Se trata, entre otras, de enfermedades tardías comunes como la hipertensión, la diabetes tipo 2, la obesidad, la oclusión de las arterias coronarias 0 , enfermedades menos frecuentes como la esquizofrenia, el trastorno bipolar o el alzheimer. (Egger, 2004, 457-460).

Una de las principales causas de las epimutaciones -la influencia materna- se produce a través de su dieta y del estrés psicológico que eventualmente sufra durante su embarazo o inmediatamente tras el parto. Entre los múltiples estudios que se han realizado a este respecto destaca uno que resulta pertinente mencionar en relación con la gestación por sustitución: se ha demostrado que la escasez nutricional en el desarrollo embrionario programa al ser humano en formación con un fenotipo epimutado gracias al cual maximizará la energía y los recursos durante el resto de su vida.

Ahora bien, si las condiciones de esa persona cambian, por ejemplo, insertándose en una sociedad donde no va a tener escasez de comida sino todo lo contrario, entonces, la programación epigenética que experimentó en su etapa embrionaria estará en desajuste con su entorno y, por ello, muy probablemente desarrollará enfermedades como la obesidad o la diabetes tipo dos (Stöger, 2008, 156-160).

En este mismo sentido, hay estudios que parecen confirmar la relación entre la dieta seguida por una madre durante su período de gestación -en particular si reduce la ingesta de alimentos que tienden a aumentar la matilación del ADN (como el ácido fólico, la metionina, la vitamina B12, la colina y la Betaína) y el padecimiento de algunas enfermedades en la edad adulta como la obesidad o la hipertensión arterial (Waterland, 2006, 401-403) o, incluso, contribuir a la aparición de 
determinados tipos de cáncer (Ross, 2003, 197-204). Más aún, el riesgo de padecimiento de estas enfermedades continuará en los descendientes de esa persona mientras no se logre revertir esa epimutación (Cooney, 2006, 1707117072).

En relación con el estado psicológico de la madre biológica, los estudios que se están desarrollando en los últimos años en este campo parecen confirmar igualmente que el mismo puede inducir cambios epigenéticos en sus descendientes y que dichos cambios afectarán a la salud futura de éstos.

De modo especial, el estrés materno -tanto en el proceso de gestación como en los primeros meses de vida del bebé tras su alumbramiento influirá en este sentido y no es baladí recordar que con frecuencia la madre biológica padece un estado de estrés (dudas, arrepentimiento, miedo) en los meses últimos de su embarazo o inmediatamente tras dar a luz en los contratos privados de gestación por sustitución (Abrams, 2015, 171-191; Robertson, 2015, 192-195) que afectará a la programación epigenética del niño así concebido aumentando, por ejemplo, el riesgo de padecer de asma años más tarde (Kozyrskyj, 2008, 142-143).

\section{CONCLUSIÓN}

El negocio global de la gestación por sustitución y el turismo reproductivo asociado al mismo requiere una regulación que ha de ser internacional si se quiere efectiva, no dando lugar a santuarios territoriales en los que puedan llevarse a cabo prácticas aberrantes que puedan desplegar efectos jurídicos en el resto de Estados. Desde el punto de vista del Derecho Internacional Privado, hasta la fecha se ha trabajado y se continua haciendo, a fin de responder a la cuestión de si un Estado estaría o no obligado a reconocer la filiación legal establecida en el extranjero de un niño nacido mediante un contrato de gestación por sustitución con sus padres de intención con los que no tiene un vínculo genético (Beaumont y Trimmings, 2016).

Siendo relevante y necesaria una aproximación jurídica a cuestiones como la filiación o la nacionalidad en conexión con la gestación por sustitución, en nuestra opinión, no es la más urgente ni la principal si nos ubicamos en un discurso centrado en la protección internacional de los derechos humanos. Pensamos por el contrario que el principio del respeto de la dignidad humana y el principio de la protección internacional de los derechos humanos de las partes más vulnerables en los contratos privados de gestación por sustitución -la mujer gestante y el niño que engendra- se convierte en referentes esenciales en la regulación internacional de la gestación por sustitución.

Desde el punto de vista de los derechos humanos, la necesidad de una regulación internacional pretende dar solución a cuestiones diversas. Por una parte, 
se invoca el derecho humano a la salud reproductiva (Casado Blanco e Ibáñez Bernáldez, 2014, 59-60), incluyendo la capacidad de procrear sin riesgos gracias a los avances en las técnicas de reproducción asistida. Por otra parte, la necesidad de una regulación internacional responde al deseo de evitar la explotación de mujeres pobres o económicamente vulnerables que se prestan a alquilar sus vientres e, incluso, a donar sus propios óvulos (Saravanan, 2015, 295-307).

Desde el discurso de los derechos humanos cada vez más voces reclaman prestar mayor atención a los derechos de los niños -la más sensible de las dos más vulnerable partes en un contrato de gestación por sustitución- en la medida en que al no existir éstos en el momento de la celebración del contrato, no se puede exigir su consentimiento, con lo cual su status se asemeja más al de "producto" resultante del mismo, con grave riesgo para su dignidad al tratar como mercancía (commodity) la vida humana.

Este riesgo se ha demostrado real en aquellos casos en los que el bebé nacido mediante esta técnica ha sido rechazado como "defectuoso" por los padres de intención (Overall, 2015, 354).

Ahora bien, salvo error por nuestra parte, hasta la fecha no se ha considerado la epigenética al hablar del interés superior del menor en el contexto de la gestación por sustitución (Agnafors, 2014, 357-363; Mcmillan, 2014, 47-55; Sifris, 2015, 396412), de ahí la pertinencia del análisis que hemos desarrollado en estas páginas centrado en el examen de los riesgos epigenéticos derivados de la gestación por sustitución para la salud del niño así concebido- y reclamando una regulación internacional desde la perspectiva de los derechos humanos para este negocio sin fronteras que sustituya el todo poderoso laissez faire, laissez passer, imperante en la actualidad.

\section{BIBLIOGRAFÍA CITADA}

Abrams, P., "The Bad Mother. Stigma, Abortion and Surrogacy", The Journal of Law, Medicine \& Ethics, 2015, Vol. 43, No. 2, pp. 171-191. Robertson, J., "Legal Change and Stigma in Surrogacy and Abortion", The Journal of Law, Medicine \& Ethics, 2015, Vol. 43, No. 2, pp. 192-195.

Agnafors, M., "The harm argument against surrogacy revisited: two versions not to forget", Medicine, Health Care and Philosophy, 2014, Vol. 17, No. 3, pp. 357363.

Beaumont, P. y Trimmings, K., "Recent Jurisprudence of the European Court of Human Rights in the Area of Cross-border Surrogacy: Is there Still a Need for 
Global Regulation of Surrogacy?", en Bagioni, G. et al., Migrant Children in the XXI Century. Selected Issues of Public and Private International Law, 2016, Editoriale Scientifica (en prensa).

Casado Blanco, M., Ibáñez Bernáldez, M., "Reflexiones legales y éticas en torno a la maternidad subrogada", Revista Española de Medicina Legal, 2014, Vol. 40, No. 2, pp. 59-60.

Cooney, G., "Germ Cells Carry the Epigenetic Benefits of Grandmother's Diet", Proc. Nat'l Acad Sci, 2006, No 103, pp. 17071-17072.

Davis, E., "The Rise of Gestational Surrogacy and the Pressing Need for International Regulation", Minnesota Journal of International Law, 2012, Vol. 21, No. 1, p. 144.

Deonandan, R., et al., "Ethical Concern for Maternal Surrogacy and Reproductive Tourism", Journal of Medical Ethics, 2012, Vol. 38, No. 12, p. 742.

Drabiak, K., et al., "Ethics, Law and Commercial Surrogacy: a Call for Uniformity", The Journal of Law, Medicine \& Ethics, 2007, Vol. 35, No. 2, pp. 300309.

Egger, G., et al., "Epigenetics in Human Disease and Prospects for Epigenetic Therapy", Nature, 2004, No 429, pp. 457-460.

Ettinger, D., "Genes, Gestation and Social Norms, Law and Philosophy: An International Journal for Jurisprudence and Legal Philosophy, 2012, Vol. 31, No. 2, pp. 243-268.

Feinberg, A., "Epigenetics at the Epicenter of Modern Medicine", 299 JAMA, 2008, p. 1348.

Gallou-Kabani, C. et al., "Nutri-epigenomics: Lifelong Remodeling of our Epigenomes by Nutritional and Methabolic Factors and Beyond", Clinical Chemistry \& Laboratory, 2007, No 45, pp. 321-323.

García Robles, R., et al., "Epignética: definición, bases moleculares e implicaciones en la salud y en la evolución humana", Revista Ciencias de la Salud, 2012, Vol. 10, № 1, p. 61.

Jirtle, R. y Skinner, M., "Environmental Epigenomics and Disease Susceptibility”, Nature Rev Genetics, Vol. 8, 2007, p. 253.

Kozyrskyj, A., et al., "Continued Exposure to Maternal Distress in Early Life is Associated with an Increased Risk of Childhood Asthma", Am J Respiratory \& Critical Care Med., 2008, No. 177, pp. 142-143.

Landman, T. y Carvalho, E., Measuring Human Rights, Routledge, NY, 2010. 
Mcmillan, J., "Making sense of Child Welfare When Regulating Human Reproductive Technologies", Journal of Bioethics Inquiry, 2014, Vol. 11, No. 1, pp. 47-55.

Overall, Ch., "Reproductive Surrogacy and Parental Licensing", Bioethics,2015, Vol. 29, No. 5, p. 354.

Reed, K. y Padkocimate, A., The Right Toolkit. Applying Research Methods in the Science of Human Rights, University of California, Berckley, 2012.

Ross, Sh., "Diet and DNA Methylation Interactions in Cancer Prevention", Ann NY Acad. Sci., 2003, No. 983, pp. 197-204.

Rothstein, M., Cai, Y., Marchant, G., "The Ghost in Our Genes: Legal and Ethical Implications of Epigenetics", Health Matrix Clavel, 2009, 19 (1), p. 5.

Sánchez Morales, H., "Impactos de la reprogenética en las tendecias demográficas y en las estructuras familiares", Arbor, 2011, Vol. 187, p. 765.

Saravanan, Sh., "Global Justice, Capabilities Approach and Commercial Surrogacy in India", Medicine, Health care and Philosophy, 2015, Vol. 18, No. 3, pp. 295-307.

Sifris, A., "The Family Courts and Parentage of Children Conceived through Overseas Commercial Surrogacy Arrangements: A Child-centred Approach", Journal of Law and Medicine, 2015, Vol. 23, No. 2, pp. 396-412.

Stöger, R., "The Thrifty Epigenotype: An Acquired and Heritable Predisposition for Obesity and Diabetes?", Bio Essays, 2008, No 30, pp. 156-160.

Waddington, C., "The Epigenotype", Endeavour, 1942, No. 1, p. 18.

Waterland, R. et al., "Maternal Methyl Supplements Increase Offspring DNA Methylation at Axin Fused", Genesis, 2006, No 44, pp.401-403.

Weidman, J. y Jirtle, R., "Epigenetic Gene Regulation: Linking Early Developmental Environment to Adult Disease", Reproductive Toxicology, 2007, No 23, p. 298. 